\title{
Avaliando a Sustentabilidade das Edificações através de Ferramentas Qualitativas e Quantitativas
}

\author{
Elisabeth M. F. Severo' ${ }^{1}$ Hipólito J. C. Sousa ${ }^{2}$ \\ eseverooo@gmail.com, hipolito@fe.up.pt \\ ${ }^{1}$ Faculdade de Engenharia da Universidade do Porto, Rua Dr. Roberto Frias, s/n 4200-465, Porto, Portugal \\ ${ }^{2}$ Faculdade de Engenharia da Universidade do Porto, Rua Dr. Roberto Frias, s/n 4200-465, Porto, Portugal
}

DOI: 10.17013/risti.19.1-14

\begin{abstract}
Resumo: Diante dos grandes impactos ambientais causados pela indústria da construção, tem-se observado o uso crescente de várias ferramentas destinadas a avaliar o ciclo de vida das construções. O objetivo deste artigo é o de apresentar e identificar as principais ferramentas qualitativas e quantitativas juntamente com seus pontos fortes e suas limitações. As qualitativas são baseadas no desempenho ambiental relativo da construção que é expresso numa única contagem final, sendo sua maior vantagem a agilidade das informações. As quantitativas são mais precisas e contam com elaboração de inventários de fluxos de materiais e de energia, não contemplando todos os aspectos ambientais avaliados devido a escassez de dados. Entretanto, há um grande consenso internacional no sentido que as metodologias qualitativas estão tomando como referência as metodologias quantitativas e que uma ferramenta pode ser perfeitamente complementar da outra.
\end{abstract}

Palavras-chave: Ferramentas Quantitativas e Qualitativas, Sustentabilidade do Ambiente Construído, Arquitetura, Engenharia Civil.

\section{Assessing the Sustainability of Buildings through Quantitative and Qualitative Tools}

\begin{abstract}
In the face of major environmental impacts caused by the construction industry, we have seen the increasing use of various tools to assess the life cycle of buildings. The purpose of this article is to present and identify the main qualitative and quantitative tools along with their strengths and limitations. Qualitative tools are based on the relative environmental performance of the building which is expressed in a single final count, and its biggest advantage the agility of the informations. The Quantitative tools are more accurate and have preparation of inventories of material and energy flows, not considering all environmental aspects evaluated due to lack of data. However, there is a large international consensus that qualitative methodologies are taking as reference the Qualitative methodologies and that a tool can perfectly complement each other.
\end{abstract}

Keywords: Quantitative and Qualitative tools, Sustainability of the Built Environment, Architecture, Civil Engineering. 


\section{Introdução}

Na segunda metade do século XX, as questões ambientais começaram a despertar preocupação em todas as áreas, envolvendo organizações não governamentais (ONG's), os governos e a sociedade em geral, especialmente em relação à indústria da construção civil, considerada como uma grande geradora de impactos ambientais, e, por essa razão, mobilizou os atores que interagem nesse contexto no sentido de estabelecer ações de conscientização, normas técnicas e leis, objetivando mitigar esses impactos. Como consequência, surgiu a necessidade de mensurar o grau de sustentabilidade das construções, através do estabelecimento de critérios e padrões que pudessem caracterizar o quão sustentável era uma edificação. Na Comunidade Européia, a partir da década de 1990, observou-se a grandeza do impacto gerado pela indústria da construção e em decorrência dessa constatação surgiram os primeiros movimentos para se ter uma construção menos impactante. Em 1997 em Helsink na Finlândia surgiu o termo Construção sustentável (EC,2001).

A construção sustentável tem o grande objetivo e desafio de desenvolver modelos e ferramentas que permitam ao setor da construção enfrentar e propor soluções aos principais problemas ambientais (Severo \& Sousa, 2016).

\section{Ferramentas de Avaliação de Edificação Sustentável}

Na década de 90, com o objetivo de caracterizar as edificações, surge na Europa, nos Estados Unidos e no Canadá várias ferramentas e métodos de avaliação de desempenho ambiental, e, posteriormente, incluindo os requisitos de sustentabilidade.

Essas ferramentas podem ser qualitativas (Certificações ou Selos) e quantitativas (Avaliação do ciclo de vida).

Muito se tem discutido sobre as vantagens de uma e de outra ferramenta, contudo, alguns autores (Costa et al, 2014) não é preciso que se use um ou outro método, de forma individualizada, é possível utilizar uma combinação dessas ferramentas, caracterizandose no que se denomina de "metodologias mistas". Acrescenta ainda Costa que "vários autores (Neri de Souza \& Costa, 2014; Venkatesh et al., 2013) defendem que existe vários fenómenos de interesse que não podem ser compreendidos unicamente por metodologias qualitativas ou quantitativas separadamente. Também acreditamos que muitas questões de investigação só podem ser plenamente respondidas pela combinação de métodos numa mesma investigação".

Por outro lado, adverte Teixeira et al (2014) que, apesar de parecer antagônico, os métodos qualitativos podem ser adotados em trabalhos de engenharia que abordam temas subjetivos ou que contenham aspectos que impossibilitem as medições, ou ainda quando as medições forem desnecessárias, podem e devem se submeter ao rigor metodológico para que se possa alcançar uma consistência nos seus resultados.

Serão apresentadas a seguir, as principais ferramentas de avaliação de sustentabilidade das edificações adotadas em vários países, inicialmente as de orientação qualitativa e, posteriormente, as quantitativas. 


\subsection{Ferramentas Qualitativas - Certificações}

A metodologia qualitativa surgiu com a finalidade de auxiliar as ciências humanas, sendo guiada por certas ideias, perspectivas e palpites relacionados ao objeto investigado, não explicitando a intenção de quantificar os resultados, sendo considerada como um método subjetivo (Comark, 1991) e (Leach, 1990).

As certificações são metodologias qualitativas que se baseiam no desempenho relativo ambiental de uma construção, quando comparada a outros edifícios e diferentes alternativas de concepção (Cole, 1998). A edificação será pontuada em vários aspectos ambientais, tais como a eficiência energética, o uso e o reaproveitamento da água e da terra, utilização de matérias-primas renováveis e materiais menos impactantes e recicláveis, conforto térmico e acústico, qualidade interna do ar, tecnologias alternativas, entre outros. As pontuações são expressas numa classificação final e podem-se basear em dados quantitativos, como por exemplo do consumo energético. A seguir descrevemse as certificações qualitativas mais utilizadas:

\section{- BREEAM (Building Research Establiment Environmental Assessment Method)}

O BREEAM é um sistema ambiental desenvolvido no Reino Unido em 1990, que define o padrão das melhores práticas da construção sustentável em escritórios e residências, abrangendo as etapas do projeto, da construção e da operação, incluindo os aspectos relacionados a energia, utilização da água, do ambiente interno (saúde e bem-estar), a poluição, transporte, materiais, resíduos, ecologia e processos de gestão. As versões contam com níveis de certificação: bom, muito bom, excelente e excepcional, e são atualizadas de acordo com os regulamentos da construção do Reino Unido. Destaca-se o Green Guide online, banco de dados disponível gratuitamente que fornece detalhes sobre os impactos ambientais no ciclo de vida de materiais e componentes da construção. Atualmente conta com 542,8 mil edificações certificadas pelo mundo e quase 2.239.400 edifícios registrados para avaliação (Breeam, 2016).

- LEED (Leadership in Energy and Environmental Design)

Sistema de certificação norte americano cuja primeira versão foi lançada em 1998 pelo plano piloto. O LEED foi desenvolvido e administrado pelo U.S. Green Building Council - USGBC, em Washington DC. Este sistema considera e avalia diversas categorias, tais como, locais sustentáveis, eficiência da água, energia, atmosfera, materiais e recursos, qualidade do ar interior (saúde humana), ambiente e inovação do projeto. As versões contam com níveis de certificação com a seguinte escala: bronze, prata, ouro e platina. Atualmente é a certificação mais popular, sendo utilizada em mais de 150 países, já certificou mais de 21 mil edificações e atualmente são mais de 72 mil processos de certificação em andamento (Leed, 2016).

\section{- GREEN GLOBES}

Certificação canadense, inicialmente baseada no Breeam. Em 2000 o sistema evoluiu para uma ferramenta "on line" de edificações, possuindo as seguintes categoriais: energia, qualidade ambiental interna, poluição (emissões), água, utilização de recursos, gestão ambiental, efluentes e outros impactos, gerenciamento de projetos, cujo destaque é a gestão de emergência (riscos nas instalações, ameaças e perigos, determina a 
prioridade da gestão de emergência). Até Março de 2016 foram certificadas mais de 1.821 edificações (Green Globes, 2016).

\section{- HQE Bâtiment}

Certificação francesa, criada em 2005 e administrada pela Association pour la Haute Qualité Environnmental, que atende também a Bélgica, Luxemburgo, Tunísia e Argélia. Este sistema em Dezembro/2015 contava com 1.645 edificações certificadas, visando melhorar a qualidade ambiental de edifícios novos e antigos com a finalidade de proporcionar estruturas seguras e confortáveis, o HQE basea-se em três componentes inseparáveis que são (HQE Bâtiments, 2016):

- Um sistema de operação de gestão ambiental (SGA), na qual o cliente define os objetivos para a operação bem como, o papel dos diferentes agentes participantes.

- Catorze metas para estruturar a resposta técnica, objetivos arquitetônicos e econômicos do cliente, ou seja, manejo de impactos ambientais exteriores (relação harmônica e imediata com o ambiente, escolha integrada dos métodos e materiais de construção, critérios de evitar o incômodo nos arredores da obra, minimização do uso de água e energia, de resíduos e da necessidade de manutenção e reparos) e a criação dum ambiente externo agradável (medidas de controle hidrotermal, controle acústico, atratividade visual, medidas de controle de odores, higiene e limpeza dos espaços internos, controle da qualidade do ar e da água;

- Indicadores de desempenho.

\section{- $\quad$ AQUA-HQE (Alta Qualidade Ambiental)}

Sistema adaptado para o Brasil em 2008, baseado no "Démarche HQE"- Bâtiment do Centre Scientifique et Technique du Bâtiment da França, pela Fundação Carlos Alberto Vanzolini, em parceria com o Departamento de Engenharia de Produção da Escola Politécnica da Universidade de São Paulo - USP. Este sistema trabalha o processo de gestão total do projeto com o objetivo de obter a qualidade ambiental do empreendimento desde a fase da concepção até a fase de uso, possuindo flexibilidade para ser aplicável a edifícios comerciais e residenciais, escolas, hotéis, na qual podem ser analisados 14 critérios para verificar a qualidade de vida do usuário, economia de água e energia, disposição de resíduos e manutenção. Até abril de 2016 foram cerificados no Brasil, 395 edifícios e 235 empreendimentos (Vanzolini, 2016).

\section{- $\quad$ LIDER A}

O Lider A é uma ferramenta portuguesa, criada em 2005, na qual objetiva: apoiar o desenvolvimento de planos e projetos voltados à sustentabilidade construtiva; avaliar e posicionar o desempenho das edificações da fase de concepção, projeto, obra e operação; suportar a gestão na fase de construção e operação, atribuir certificação pela avaliação independente e servir como um instrumento de mercado que distingui e valoriza os empreendimentos. Até Julho de 2016 contava com 34 certificações em empreendimentos portugueses (LiderA, 2016).

Na Tabela 01, a seguir, é apresentado um comparativo dos pontos fracos e fortes dos principais sistemas de certificação. 


\begin{tabular}{|c|c|c|}
\hline $\begin{array}{l}\text { SISTEMAS DE } \\
\text { CERTIFICAÇÃO }\end{array}$ & PONTOS FRACOS & PONTOS FORTES \\
\hline BREEAM & $\begin{array}{l}\text { - Requisitos muito exatos } \\
\text { - Sistema de ponderação complexo } \\
\text { - Perfil de mercado } \\
\text { - Custos do processo elevados para } \\
\text { a certificação }\end{array}$ & $\begin{array}{l}\text { - Permite a avaliação e comparação c/ } \\
\text { diferentes edifícios } \\
\text { - Auditoria independente } \\
\text { - Ajustado a cultura e legislação do } \\
\text { Reino Unido } \\
\text { - Pode avaliar qualquer edifício com a versão } \\
\text { Bespoke } \\
\text { - Há um maior peso para os indicadores } \\
\text { "muito importante" e "importante", quando } \\
\text { comparado ao LEED }\end{array}$ \\
\hline LEED & $\begin{array}{l}\text { - Baseado em sistemas americanos } \\
\text { - É exigido uma intensa } \\
\text { documentação } \\
\text { - Nenhuma auditoria } \\
\text { independente } \\
\text { - Difícil de avaliar a função e a } \\
\text { forma separadamente }\end{array}$ & $\begin{array}{l}\text { - Forte Marketing } \\
\text { - Muita informação disponível } \\
\text { - Não há a necessidade de treinar assessores }\end{array}$ \\
\hline $\begin{array}{l}\text { GREEN GLOBES } \\
\text { (Baseado no } \\
\text { BREEAM) }\end{array}$ & $\begin{array}{l}\text { - Para acessar o sistema on-line é } \\
\text { necessário registro como usuário, } \\
\text { definir forma de pagamento e } \\
\text { só após é permitido o acesso ao } \\
\text { questionário }\end{array}$ & $\begin{array}{l}\text { - Os itens são avaliados separadamente, } \\
\text { prevalecendo o enfoque ambiental }\end{array}$ \\
\hline HQE Bâtiment & $\begin{array}{l}\text { - Sistema ainda encontra } \\
\text { certa oposição e crítica entre } \\
\text { alguns projetistas que temem } \\
\text { que a normalização conduza } \\
\text { a construção a projetar } \\
\text { impulsionada pelas exigências de } \\
\text { certificação }\end{array}$ & $\begin{array}{l}\text { - O sistema identifica } 14 \text { questões ambientais } \\
\text { e abrange } 2 \text { aspectos: qualidade ambiental } \\
\text { do edifício e a gestão do projeto inteiro } \\
\text { - A técnica do sistema, agora já está bem } \\
\text { conhecida pelos profissionais franceses } \\
\text { - Rotulagem verde para edifícios apoiada } \\
\text { pelo governo francês } \\
\text { - Todas as fases são consideradas para o } \\
\text { método e resultado da avaliação } \\
\text { - Certificação disseminada em conferências, } \\
\text { treinamentos, programas e publicações }\end{array}$ \\
\hline $\begin{array}{l}\text { AQUA } \\
\text { (Baseado no HQE } \\
\text { Bâtiment) }\end{array}$ & $\begin{array}{l}\text { - Falta de divulgação do sistema } \\
\text { - Dificuldades na obtenção das } \\
\text { informações } \\
\text { - Altos custos do processo } \\
\text { - Ainda com pouca } \\
\text { representatividade }\end{array}$ & $\begin{array}{l}\text { - Adaptada para o contexto brasileiro } \\
\text { - Avaliação de maneira evolutiva (categorias, } \\
\text { subcategorias e preocupações) } \\
\text { - Equipe consultora que aplica questionário }\end{array}$ \\
\hline
\end{tabular}

Tabela 1 - Comparativo dos Pontos Fortes e Fracos dos Principais Sistemas de Certificação Elaborado a partir de Yüce, (2012); Parker,(2009) e (Bueno \& Rossignolo,(2007).

\subsection{Ferramentas Quantitativas}

Conforme Burns \& Grove, (1987) e Corner, (1991) a metodologia quantitativa apareceu como ferramenta para auxiliar as ciências exatas e antecede a qualitativa. A ferramenta quantitativa tem a preocupação com a investigação de coisas com a finalidade de medir através de dados numéricos, de forma objetiva. Esse processo descreve, testa e analisa causas e relações de efeito. Os métodos quantitativos se utilizam de experimentos 
verdadeiros e trazem grande contribuição ao conhecimento universal, com respostas científicas (Melia, 1982).

Entretanto, Gonçalves \& Duarte, (2006) e Kientzel (2010), dizem que há uma grande necessidade de se fazer a avaliação, devido ao grande impacto produzido nos processos construtivos. Para tal, a Comunidade Europeia, Estados Unidos, Canadá e alguns países asiáticos trabalharam suas legislações einvestiram nas certificações de projeto e de construção. No Brasil a medição qualitativa é realizada através dos selos AQUA, LEED entre outros, porém apesar dos esforços metodológicos e científicos, ainda falta uma legislação ampla e específica para o país no sentido de se ter uma avaliação de todo o ciclo de vida das construções.

\subsection{Avaliação do Ciclo de Vida - ACV}

De acordo com a Society of Environmental Toxicology and Chemistry - SETAC (1993), uma ACV é um processo que tem o objetivo de avaliar as cargas ambientais decorrentes de uma atividade, processo ou produto na qual identifica e quantifica todos os impactos sobre o meio ambiente, ou seja, contaminações, degradações e geração de resíduos, medindo os impactos através de indicadores; e por fim analisa-se as possíveis melhorias do ciclo como um todo, do início (berço) até o final túmulo), incluindo a reutilização ou reciclagem.

A série ACV: ISO 14040 normas internacional, foi criada devido ao número crescente de impactos ambientais. Inicialmente avaliava a quantidade de materiais, energia e resíduos e depois foi utilizada na avaliação ambiental da produção e os bens de consumo (USEPA, 2012).

Conforme a ABNT NBR ISO 14.040:2009 a ACV possui cinco etapas básicas, conforme descritas e observadas na Figura 01 a seguir.

- Definição dos objetivos e abrangência

- Análise de inventário

- Avaliação dos impactos

- Interpretação

- Análise de melhorias considerando as limitações do estudo da ACV

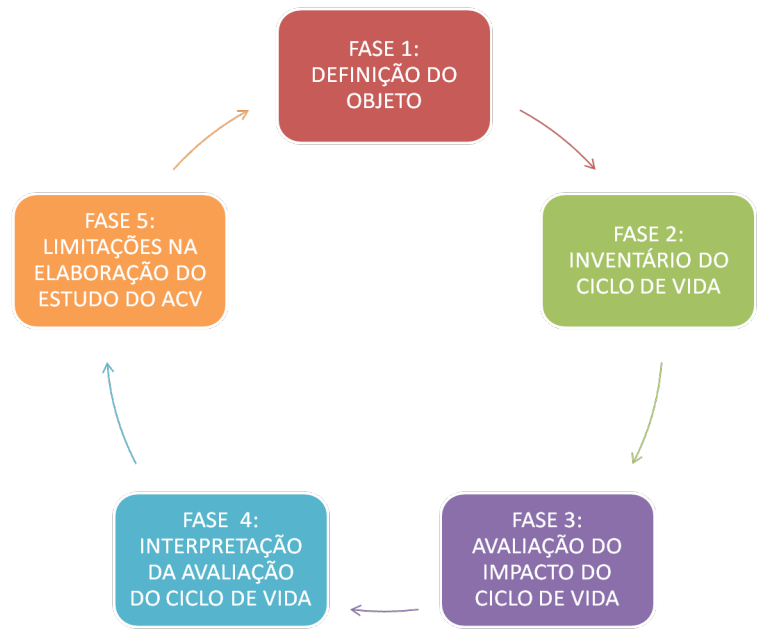

Figura 1 - Fases da ACV (Fonte: ABNT NBR ISO 14.040:2009) 
A avaliação do ciclo de vida (ACV) é uma ferramenta quantitativa e analítica. Na qual há a definição do seu objetivo e escopo, da realização de um levantamento quantificado de dados de entrada, saídas e dos impactos ambientais potenciais de um determinado produto ou serviço ao longo do seu ciclo de vida; e dá interpretação dos resultados com a indicação de melhorias.

A fase de definição determina o limite do sistema a ser estudado, considerando-se a qualidade dos dados necessários para a aplicação da ACV (Reis, 1995; Carvalho Filho et al 1997; Grigoletti, 2001).

Na fase de Análise de Inventário são feitas as compilações e quantificações das entradas e saídas, sendo essas representadas por um fluxograma onde são determinados os limites do sistema a ser estudado (UNEP, 1996 apud Grigoletti, 2001).

Na Avaliação dos Impactos que abrange a Classificação, a Caracterização e a Valoração; tem a finalidade de analisar os resultados através da classificação, caracterização e avaliação dos impactos ambientais dentro de limites definidos nos objetivos e abrangência (Reis,1995).

A classificação é composta de:

- Recursos: energia, materiais, água e solo;

- Danos ao ecossistema: aquecimento global, redução da camada de ozônio, chuva ácida, formação de foto-oxidantes, impactos ecotoxicológicos, alterações de habitat e impactos a biodiversidade;

- Saúde humana: impactos tóxicos e não tóxicos.

A caracterização é a quantificação por categorias das entradas e saídas.

A valoração procura comparar a importância relativa das diferentes categorias de impactos individuais para o sistema em estudo onde são refletidos os valores sociais e preferências.

Na fase da interpretação há a interpretação de forma contínua, permitindo assim, a tomada de decisões.

Na fase da análise de melhorias, conforme Reis (1995), os principais benefícios são:

- Identificar as melhores oportunidades para o aprimoramento ambiental do sistema;

- Evitar decisões que apenas transfiram o ônus ou impactos ambientais de um sistema para outro; e

- Realizar comparações entre sistemas similares.

A Figura 02 a seguir, mostra o fluxo da ACV de uma edificação (Severo, 2013).

$\mathrm{Na}$ ACV todas as medidas são indicadores de cargas ambientais, resultantes da exploração de matérias-primas, da fabricação, do transporte de materiais, do uso e descarte do produto (ISO, 2005).

Na fase da definição determina-se o limite do sistema a ser estudado, bem como a qualidade dos dados a serem aplicados na ACV. No inventário são feitas as compilações 


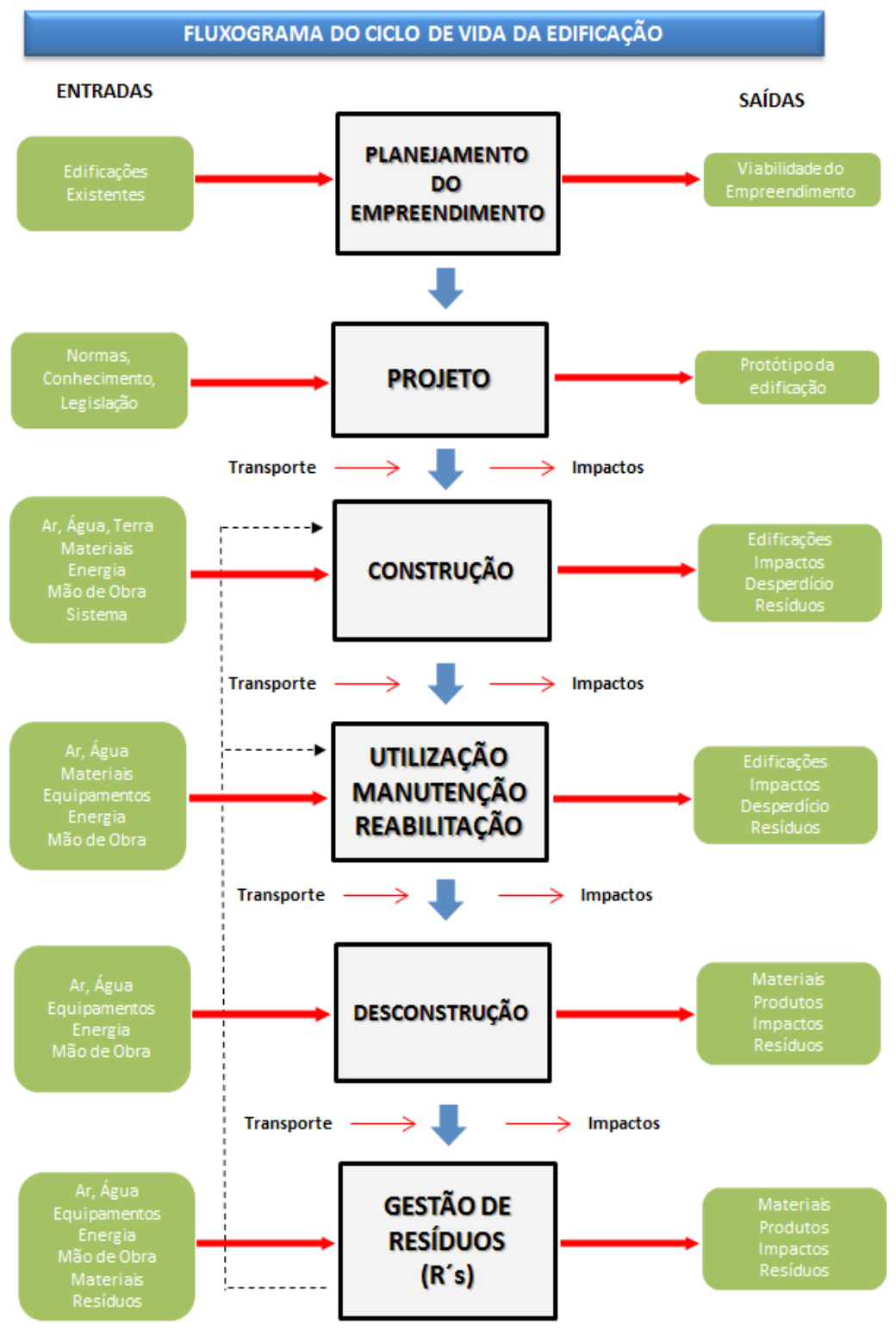

Figura 2 - Fluxograma do Ciclo de Vida da Edificação (Adaptado de ISO PD TS 21.931, 2005).

e quantificações de entradas e saídas, apresentadas num fluxograma onde determinase os limites a serem estudados. A avaliação dos impactos abrange a classificação, a caracterização e a valoração, com o objetivo de analisar os resultados (Gricoletti, 2001 ). 


\subsection{Principais Benefícios e Limitações da Avaliação do Ciclo de Vida (ACV)}

São vários os benefícios da metodologia ACV. Com a utilização da ferramenta ACV é possível analisar os impactos ambientais causados por sistemas, produtos, processos ou atividades.

Permite também aos seus utilizadores:

- Desenvolver o planejamento estratégico do projeto do produto e ou processo;

- Analisar os balanços (ganhos/perdas) ambientais associados com um ou mais produtos/processos específicos de modo a que os visados (estado, comunidade, etc.) aceitem uma ação planejada;

- Quantificar as descargas ambientais para o ar, água, e solo relativamente a cada estágio do ciclo de vida e/ou processos, dando suporte a auditoria ambiental;

- Identificar e solucionar problemas para a melhoria do produto/processo, bem como gera informações ao consumidor;

- Identificar oportunidades de melhorias ambientais, visando a redução dos impactos ecológicos e na saúde humana;

- Viabilizar os selos ecológicos a as certificações de produtos ou serviços;

- Identificar impactos em uma ou mais áreas ambientais específicas de interesse;

- Incorporar o ACV no projeto de produtos incluindo a seleção de materiais, de processos de produção e nas etapas de pós-uso (reabilitação, reciclagem e reaproveitamento de materiais e produtos e desativação);

- As limitações são a grande quantidade de dados necessários para obter-se o processamento dos diversos sistemas e subsistemas. Portanto é uma ferramenta que ainda tem certas limitações de custo, tempo e operacionais devido a grande massa de dados a ser trabalhada (Rodrigues, Zoldan, Leite \& Oliveira, 2008).

\subsection{Sistemas Computacionais para ACV}

No estudo de ACV é grande a quantidade de dados necessários para obter-se o processamento da análise, sendo que um inventário de ciclo de vida duma edificação é composto de vários inventários de diversos sistemas e subsistemas. É uma técnica que ainda tem certas limitações operacionais devido ao grande volume de dados a ser trabalhado (Rodrigues et al, 2008).

Para se avaliar o ciclo de vida de um produto é necessário ter acesso a informações sobre os impactos ambientais em todas as etapas desde a extração e produção das matériasprimas, produtos e sistemas bem como de todos os insumos utilizados no processo (energias, água, solo, ar e outros).

No sentido de auxiliar e agilizar os processos que envolvem uma ACV foram desenvolvidos softwares para permitir a trabalhabilidade de processamento de um grande volume de dados de maneira a apresentar uma maior confiabilidade das informações geradas pelos os resultados dos relatórios. O software é fundamental pois, minimiza o tempo, permite a simulação da ACV de produtos e processos, prevê os impactos ambientais, de modo a embasar o planejamento e a tomada de decisão. 
Em geral, há uma quantidade razoável de softwares disponíveis, sendo que a maioria é comercializada para instalação no equipamento do usuário, existindo, porém, alguns softwares cujo acesso é feito exclusivamente na forma online, diretamente através de acesso ao website do titular do software, dentre os quais se destacam o BEES, o ATHENA e o SB Tool.

A avaliação do ciclo de vida (ACV) não é uma ferramenta originalmente desenvolvida para edificações ou projetos complexos de grande porte, mas já há ferramentas ACV como por exemplo o software BEES 4.o que faz a comparação dos produtos utilizados nas fases de especificação e de aquisição e o software ATHENA Environmental Impact Estimator (EIE) que faz a análise de parte e de todo o edifício desde a concepção do projeto, trazendo uma série de indicadores de impactos para as etapas de projeto, aquisição dos materiais e na construção da edificação. Versões regionais estão em desenvolvimento na National Association of Home Builders que trabalhará com a atribuição de pontos para cada categoria de impacto, permitindo informações estratégicas para a melhor tomada de decisão nas fases de concepção e projeto (Carmody \& Trusty, 2005).

A maioria dos sistemas não consegue esclarecer as diferenças entre o desempenho ambiental e o desempenho real (potencial) do edifício em operação (Sidoni, 2004).

Atualmente o único sistema que apresenta todas as aplicações potenciais é o SB Tool, que permite aos utilizadores editar dados de acordo com as diferentes tecnologias, prioridades, estilos construtivos e até valores culturais, sendo aplicável em qualquer tipo de edificação, ou seja, residencial, comercial, industrial, e outros, em qualquer região do mundo. Projetistas, construtores e outros envolvidos na edificação fornecem a descrição do edifício, mas não a definição dos benchmarks ou dos fatores de ponderação, sendo necessário a inserção de um grande volume de dados relativos ao contexto onde o empreendimento está localizado (Librelotto \& Jalali, 2008). O sistema pode ser utilizado também por organismos autorizados, tais como municípios e organizações não-governamentais (ONGs) para estabelecer sistemas de classificação de acordo com suas próprias regiões e tipos de construção. Permite uma vasta parametrização de questões relativas a construção sustentável, podendo ser estabelecido entre 10 a 115 critérios (Larsson, 2015).

São tratadas pelo SB Tool as seguintes questões de performance:

1. Alterações climáticas

2. Destruição da camada de ozono da estratosfera

3. Acidificação dos recursos terrestres e aquáticos

4. Eutrofização das massas de água

5. Criação de ozono fotoquímico (POCP)

6. Mudanças na biodiversidade local

7. O esgotamento da energia primária não renovável;

8. O esgotamento dos outros do que de energia primária recursos não renováveis;

9. O esgotamento dos recursos de água doce não-renováveis 
10. O esgotamento dos recursos da terra com valor ecológico ou agrícola

11. Esgotamento dos depósitos de resíduos sólidos adequados para resíduos não perigosos

12. Riscos de eliminação ou armazenagem de resíduos perigosos não-radioativo

13. Riscos de eliminação ou armazenagem de resíduos radioactivos

14. Capacidade dos usuários com deficiências funcionais na utilização dos recursos

15. Proteção e segurança dos usuários pessoais

16. Saúde, bem-estar e produtividade para os usuários de instalação

17. Saúde, segurança e bem-estar da população off-site local

18. Alterações sistemas sociais ou culturais locais

19. Riscos ou benefícios financeiros para os investidores

20. Viabilidade da Habitação para comércio varejista

21. Mudanças no sistema económico local (emprego, estímulo econômico)

O SB Tool tem sido utilizado como base para o desenvolvimento de vários sistemas de classificação nacional como por exemplo em Portugal (SB Tool PT), República Checa (SB Tool CZ), Itália (Protocolo ITACA) e Espanha (Verde).

Tanto as ferramentas qualitativas (certificações) como as quantitativas (ACV) estão em constante evolução e as mesmas devem ser atualizadas quanto á abrangência e a compatibilidade de suas aplicações numa determinada edificação.

\section{Conclusões}

É fundamental a adoção de ferramentas de avaliação para as indústrias, em especial para o setor da construção devido ao grande impacto ambiental no seu ciclo de vida.

Avaliar o ciclo de vida das edificações é uma tarefa complexa, mas, extremamente importante devido ao grande impacto ambiental, na qual envolve vários materiais, produtos e serviços.

Verifica-se uma rápida evolução das ferramentas qualitativas (certificações) e quantitativas (ACV) no sentido de criar uma avaliação voltada a um ambiente construído sustentável.

Na ausência de dados para compor uma ACV, recomenda-se utilizar a avaliação qualitativa (certificações) que dá uma boa noção do viés sustentável.

Apesar das metodologias serem diferentes pois a qualitativa qualifica e a quantitativa quantifica, uma não é superior a outra pois, ambas têm pontos fortes e limitações e devem preferencialmente ser utilizadas em conjunto. 
Com a adoção de ferramentas de ACV na composição das ferramentas qualitativas (certificações) trarão um grande avanço, contribuindo com a integração contínua de ACV possibilitando detectar-se os impactos ambientais de modo mais abrangente e significativo para a sustentabilidade das edificações.

Com a criação de banco de dados regionais, haverá uma maior facilidade para a ferramenta softwares ACV que dá uma maior precisão, possibilitando detectar os impactos ambientais de maneira mais detalhada, o que contribuíra de maneira significativa para o processo sustentável das edificações.

\section{Referências}

ABNT. (2010). Associação Brasileira de Normas Técnicas. ABNT NBR ISO 14040:2009. Disponível em:www.abntcatalogo.com.br/norma.aspx?ID=1190 Acesso em 06 Mar 2010.

Breeam. (2016) Building Research Establishment Environmental Assessment Method. o6-jan-2016. Sítio Web: http://www.breeam.com/.

Bueno, C.; Rossignolo, J.A. (2007). Desempenho Ambiental de Edificações: Cenário Atual e Perspectivas dos Sistemas de Certificação. Pesquisa e Tecnologia Minerva, 45-52. São Paulo, SP. 13-mar-2013. Sítio web: http://www.fipai.org.br/Minerva\%20 07(01)\%2006.pdf.

Burns, N., Grove, S.K. (1987). The practice of research, conduct, critique, and utilization. Philadelphia. Saunders.

Carmody, J.; Trusty, W. L. (2005). Life Cycle Assessment Tools. Research Informe Design of the University of Minnesota, 5(3), 1-5.

Cole, R.J. (1998). Charting the Future: Emerging Trends in Building Environmental Assessment Methods. School of Architecture, University of British Columbia. Building Research and Information, 26(1), 3-16. Doi: 10.1080/096132198370065

Comark, D.S. (1991). The research process. Oxford. Black Scientific.

Costa, A. P.; Reis, L. P.; Neri de Souza, F. (2014). Investigação Qualitativa para Sistemas e Tecnologias de Informação. RISTI - Revista Ibérica de Sistemas e Tecnologias de Informação, 2014(E2), ix-xii. Doi: 10.17013/risti.e2.ix-xii

DGNB. (2016). Deutsche Gesellschaft fur Nachhaltiges Bauen. 05-abr-2016. Sítio web: http://www.dgnb-system.de/en/.

EC-European Commission. (2001). Competitiveness of the Construction Industry. Agenda for Sustainable Construction in Europe. Brussels.

Gonçalves, J.C.S., Duarte, D.H.S. (2006). Arquitetura Sustentável: uma integração entre ambientes, Projeto de Pesquisa, Prática e Ensino. Ambiente Construído. 6(4), p.51-81.

Green Globes. (2016). The Practical Building Rating System. 30-abr-2016. Sítio web: http://www.green globes.com. 
Gricoletti, G. C. (2001). Características de Impactos Ambientais de Indústrias de cerâmica vermelha do Estado do Rio Grande do Sul. Porto Alegre. UFRGS.

HQE Bâtiment. (2016). Haute Qualité Environnementale. 13-jan-2016. Sítio web: http//:assohqe.org/hqe/.

ISO. (2005). TC59/SC17/WG4. Sustainability in building construction: framework for methods of assessment for environmental performance of construction works. Part 1: Buildings. ISO PDTS 21.931.Geneva.

Kientzel, J. (2010). Voluntary Environmental Building Rating Schemes as a Method of Sustainable Public Procurement. Maastricht University/Union National University (UNI-MERITY), Seminary Sustainable Procurement of the Stockholm University. Netherlands.

Kohler, N. (1999). An observer's perspective on the relevance of the Green Building Challenge, Building Research and Information, 27(4), Green Building University.

Larsson, N. (2015). SBTool for 2015. International Initiative for a Sustainable Built Environment. Maio 2015. Sítio web: http://www.iisbe.org/system/files/ SBTool\%20Complete\%2004May15.pdf.

Leach, M. (1990). Philosophical choice. Journal of Education, 3(3), 16-18. John Wiley \& Sons, Inc. USA.

Librelotto, D., Jalali, S. (2008). Aplicação de uma ferramenta de Análise do Ciclo de Vida em Edificações Residenciais- Estudo de Caso. Universidade do Minho, Guimarães, PT.

Leed. (2016). Leadership in Energy and Environmental Design. 05-mai-2016. Sítio web: http://www.usgbc.org/certification.

Lider A. (2016). Sistema de Avaliação da Sustentabilidade. 22-abr-2016- Sítio web: http://www.lidera.info.

Melia, K.M. (1982). Qualitative Methodology. Journal of Advanced Nursing, 7(4), 327-335.

Parker, J. (2009). Breeam or Leed - strengths and weaknesses of the two main environmental assessment methods. England, UK. BSRIA Ltda.

Rodrigues, C.R.B.; Zoldan, M.A.; Leite M.L.G.; Oliveira,I.L. (2008). Sistemas Computacionais de Apoio e Ferramentas de Análise de Ciclo de Vida do Produto $(A C V)$. XXVIII Encontro Nacional de Engenharia de Produção. Rio de Janeiro, Brasil.

SETAC. (1993). A conceptual Framework for Life-Cycle Impacts Assessment- Guidelines for Life-Cycle Assessment: A "Code of Practice"- Society for Environmental Toxicology and Chemistry-Bruselas.

Severo, E.; Sousa, H. (2016). Ferramentas Quantitativas e Qualitativas para a Avaliação da Sustentabilidade das Edificações. Atas- Invetigação Qualitativa em Engenharia e Tecnologia do CIAIQ 2106- Vol. 4 - ISBN: 978-972-8914-61-5. 
Severo, E. M. F. (2013). Sustentabilidade nas Edificações Residenciais no Brasil. PTIProjeto de Tese de Investigação apresentado ao PRODEC-Programa Doutoral de Engenharia Civil da FEUP- Faculdade de Engenharia da Universidade do Porto, PT.

Sidoni, R. C. (2004). Avaliação da Sustentabilidade da Construção. Relatório de Projecto Individual, Escola de Engenharia da Universidade do Minho.

Teixeira, F. R.; Mayr, L. R.; Paisana, A. V.; Vieira, F. D. (2014). Escolhas metodológicas em investigação científica: aplicação da abordagem de Saunders no estudo da influência da cultura na competitividade de clusters. RISTI - Revista Ibérica de Sistemas e Tecnologias de Informação 2014(E2), 85-98. Doi:10.17013/risti.n.85-98.

USEPA. (2012). ISO 14.040. United States Environmental Protection Agency. USA. 21-fev-2012- Sítio web: http://nepis.epa.gov/Exe/ZyPURL.cgi?Dockey=30004 RLB.txt.

Udo, Helias de Hans A. (2001). The Dutch Building Industry. Part II: A Challenge for LCA. Journal of Industry Ecology 5(1): 4-6. Doi: 10.1162/108819801753358463

Vanzoline.(2016). Certificado Aqua-HQE. 03-mai-2016. Sítio web: http://www. vanzolini.org.br/hotsite-aqua.asp

Yüce, M. (2012). Sustainability Evaluation of Green Building Certification Systems. Thesis of Master of Science. Florida International University. Miami, Florida, USA. 21-out-2012. Sítio web: http://digitalcommons.fiu.edu/cgi/ viewcontent. cgi article $=1882 \&$ context $=$ etd 